\section{Polypoidal choroidal vasculopathy and history of central serous chorioretinopathy}

\begin{abstract}
Purpose To evaluate the possible causative role of central serous chorioretinopathy (CSC) in the development of exudative age-related macular degeneration (AMD). Methods In a cross-sectional study at an institutional setting, 150 control subjects who had senile cataract or nasolacrimal duct stenosis and who were older than 50 years were enrolled. The background data for 89 patients with typical AMD (tAMD) and 138 patients with polypoidal choroidal vasculopathy (PCV) were used for comparison. Their medical records were taken for history of CSC, hypertension, systemic steroid use, and smoking. The fundus was also evaluated for signs of atrophic retinal pigment epithelial (RPE) tract and for focal photocoagulation scars in the macula.
\end{abstract}

Results After adjusting for age, gender, and history of hypertension, systemic steroid use, and smoking, history of CSC was significantly more frequent $(P<0.0001)$ in patients with PCV (15 patients, 10.9\%)

Department of Ophthalmology, University of Tokyo School of Medicine, Tokyo, Japan

Correspondence: T Ueta, Department of Ophthalmology, University of Tokyo School of Medicine, 7-3-1 Hongo, Bunkyo-ku, Tokyo 113-8655, Japan Tel: +8133815 5411: Fax: +81338170798. E-mail: ueta-tky@umin.ac.jp

Received: 22 July 2013 Accepted in revised form: 1 May 2014 Published online: 13 June 2014 compared with patients with tAMD

(2 patients, $2.2 \%$ ) or control subjects (0 patients). On fundoscopy, an atrophic RPE tract (seven patients) or a focal photocoagulation scar (one patient) was observed only in patients with PCV (eight patients, $5.8 \%$ ), and the frequency was statistically significant compared with that with tAMD $(P=0.0143)$ or control subjects $(P=0.0143)$. The laterality of CSC and AMD involved the same eye in 9 of $\mathbf{1 0}$ patients among those who had unilateral AMD and a reported unilateral CSC history.

Conclusion A history of CSC may be a predisposing factor for the development of PCV in the Japanese population. Eye (2014) 28, 992-997; doi:10.1038/eye.2014.132; published online 13 June 2014
Introduction

Exudative age-related macular degeneration (AMD) is a major cause of legal blindness worldwide. ${ }^{1,2}$ One of the major critical differences in exudative AMD between the Asian population, including the Japanese, and the Western population is an increased frequency of polypoidal choroidal vasculopathy (PCV) in the former. ${ }^{3} \mathrm{PCV}$ is considered to be a distinct clinical entity and was first reported approximately two to three decades ago. ${ }^{4}$ Through broadening recognition of PCV as a distinct disorder and improvements in the resolution of indocyanine green angiography (ICGA), PCV may now involve at least half of the patients with exudative AMD in the Japanese population. ${ }^{3}$ Compared with typical AMD (tAMD), PCV shows slower progression, ${ }^{5-7}$ better visual prognosis despite more chronic persistence, ${ }^{5-7}$ better therapeutic response to photodynamic therapy, ${ }^{8}$ less responsiveness to anti-vascular endothelial growth factor (VEGF) therapy, ${ }^{9}$ and thicker choroid. ${ }^{10,11}$ In contrast, PCV and tAMD have genotypic similarities at the AMD-associated loci. $^{12}$

In our previous study, ${ }^{13}$ we compared background factors between patients with tAMD and those with PCV. Although tAMD and PCV were similar in most of these factors, we observed that the history of central serous chorioretinopathy (CSC) was more frequent in patients with PCV than in those with tAMD. Other researchers have also recently substantiated the frequency of CSC in patients with PCV. ${ }^{14,15}$ However, CSC as a predisposing factor for the development of PCV has remained unclear because the issue could not be addressed without the evaluation of the history of CSC in patients with PCV and non-AMD 
control subjects. The objective of the present study was to examine whether the history of CSC could be a predisposing factor for the development of PCV or tAMD by evaluating control subjects.

\section{Materials and methods}

\section{Participants}

The present study was conducted with the approval of the Institutional Review Board of the University of Tokyo Hospital, and all patients enrolled in the study provided informed consent.

The background data of patients with exudative AMD (ie, either tAMD or PCV) in our previous study ${ }^{13}$ was used for comparison with the enrolled control subjects. In this study, we gathered background data for control subjects who were both older than 50 years and without exudative AMD. In other words, among the patients who visited our general ophthalmology outpatient clinic, patient with either senile cataract or nasolacrimal duct stenosis (NDS) and those who were older than 50 years and without exudative AMD were included in the present study. Males and females were recruited in a ratio of approximately $2: 1$ to approximate the male/ female balance of the AMD group.

The survey to gather data from control subjects was conducted from October 2010 through March 2012. All enrolled patients were mentally able to answer the questions about their medical history.

For patients with exudative AMD, subtype diagnosis of either tAMD or PCV was based on the findings of both the fluorescein angiography and ICGA; ICGA has been critically important in the diagnosis of PCV. In this study, data on 227 patients with exudative AMD was used.

\section{Data collection}

Background data of subjects in the control group was collected using the same questionnaire as that used in our previous study, ${ }^{13}$ as well as through the patients' medical records. A history of CSC was the primary concern in the present study; however, significant confounders such as a history of systemic steroid use, hypertension, and smoking were also considered. Subjects were interviewed about their history of CSC; if the answer was yes, laterality of the condition was also requested.

Apart from investigations regarding the history, fundus findings were examined. Findings indicating a history of CSC such as an atrophic retinal pigment epithelial (RPE) tract ${ }^{16}$ or focal photocoagulation scars in the macula were evaluated in control subjects.

\section{Investigated background factors}

The factors investigated in the present study were gender, age, smoking history by pack-years, a history of CSC, a history of systemic use of steroids, and a history of hypertension. The last two factors were wellrecognized major risk factors for CSC. ${ }^{17}$ The presence of CSC episodes in one or both eyes was considered as a positive response in the history. If a reported CSC episode occurred when the subjects were $\geq 50$ years old and within 10 years of the diagnosis of exudative AMD, the episode was not regarded as CSC in this study. Hypertension was defined as repeated systolic blood pressure measurements of $140 \mathrm{~mm} \mathrm{Hg}$ or more, diastolic blood pressure measurements of $90 \mathrm{~mm} \mathrm{Hg}$ or more, or the use of antihypertensive medication at the most recent annual check-up. The history of systemic use of steroid was investigated.

\section{Statistics}

The statistical analysis was performed with JMP Pro 10.0.2 software (SAS Inc., Cary, NC, USA). Univariate analyses were performed with Student's $t$-test or ANOVA and the chi-square test. A post hoc Marascuilo procedure was used to evaluate the statistical significance between each pair of the three groups (ie, control, tAMD, and PCV). Multivariate analysis was performed through a logistic regression model. A $P$ value of $<0.05$ was considered statistically significant.

\section{Results}

The study population of the primary survey consisted of 227 exudative AMD patients (89 with tAMD and 138 with PCV) and 150 control participants (42 with NDS and 108 with senile cataract). In the control group, no patients had fundal pathologic findings, except two patients with non-proliferative diabetic retinopathy.

Table 1 shows the distributions and proportions of gender, age, and histories of CSC, hypertension, and systemic steroid use. A history of CSC that was reported in two patients with PCV was not included because the episodes supposedly occurred after the age of 50 years and within 10 years after the onset of AMD. There was no difference between the control, tAMD, and PCV groups among age, gender, and histories of hypertension and systemic steroid use based on univariate and multivariate analyses. In contrast, smoking $>10$ packyears was more frequently observed in the tAMD and PCV groups compared with the control group. No participant in the control group reported a history of CSC, while 2 patients (2.2\%) with tAMD and 15 patients (10.9\%) with PCV reported a history of CSC $(P<0.0001$, by univariate or multivariate logistic regression model). 
Table 1 Comparison of a history of CSC and other key background factors among patients with tAMD, those with PCV and the control subjects

\begin{tabular}{lccccc}
\hline & Control $(\mathrm{n}=150)$ & tAMD $(\mathrm{n}=89)$ & $P C V(\mathrm{n}=138)$ & P-value (univariate) & P-value (multivariate) \\
\hline Age, mean (SD) & $71.2(8.2)$ & $70.8(8.8)$ & $70.5(7.4)$ & 0.60 & 0.76 \\
Male, $n$ (\%) & $105(70)$ & $70(78.7)$ & $105(76.1)$ & 0.27 & 0.50 \\
CSC, $n$ (\%) & $0(0.0)$ & $2(2.3)$ & $15(10.9)$ & $<0.0001$ & $<0.0001$ \\
Systemic steroid, $n(\%)$ & $9(6.0)$ & $2(2.3)$ & $5(3.6)$ & 0.34 & 0.44 \\
Hypertension, $n$ (\%) & $65(43.3)$ & $44(49.4)$ & $57(41.3)$ & 0.47 & 0.64 \\
Smoking ( $>10$ pack-years), $n(\%)$ & $58(38.7)$ & $60(60.1)$ & $83(60.1)$ & $<0.0001$ & $<0.0001$ \\
\hline
\end{tabular}

Abbreviations: CSC, central serous chorioretinopathy; PCV, polypoidal choroidal vasculopathy; tAMD; typical age-related macular degeneration.

As shown in Figure 1, compared with the control group, the frequency of a history of CSC was not significantly higher in the tAMD group $(P=0.36$, by chi-square test and post hoc Marascuilo procedure), whereas it was significantly higher in the PCV group $(P=0.0002$, by chi-square test and post hoc Marascuilo procedure). A history of CSC was more frequent in the PCV group than in the tAMD group, as shown in our previous study $^{13}$ ( $P=0.0286$, by chi square test and post hoc Marascuilo procedure).

The mean \pm SD interval for the onset of CSC and AMD was $28 \pm 13$ years (range; $8-61$ years). The age of presentation for exudative AMD with or without a CSC history was similar (68.6 and 70.6 years, respectively; $P=0.33$, by Student's $t$-test). Patients with PCV who had a history of CSC visited our clinic at an earlier mean age of $67.9 \pm 7.4$ years compared with other patients with PCV (70.7 \pm 7.4 years); however, this difference was not statistically significant ( $P=0.17$, by Student's $t$-test). Of the 17 patients with a history of CSC, 10 were patients with unilateral exudative AMD who reported a history of unilateral CSC. Nine of the 10 patients reported that CSC had been in the same eye as that had the exudative AMD.

Subsequently, we investigated for fundus findings such as an atrophic RPE tract or a focal PC scar in the macula, indicating a history of CSC (Figures 2 and 3). Atrophic RPE tracts were identified in patients with PCV (7/138 patients), but not in patients in the control group or in those with tAMD. A focal PC scar in the macula was identified only in one patient with PCV. When the two findings were combined, these objective findings were more frequently identified in the PCV group $(8 / 138$ patients $(5.8 \%)$ compared with the control group $(0 / 150$ patients, $P=0.0143$, by chi-square test and post hoc Marascuilo procedure) or with the tAMD group (0/89 patients, $P=0.0143$, by chi square test and post hoc Marascuilo procedure).

\section{Discussion}

The present study enrolled non-AMD control subjects and confirmed that a history of CSC was more common

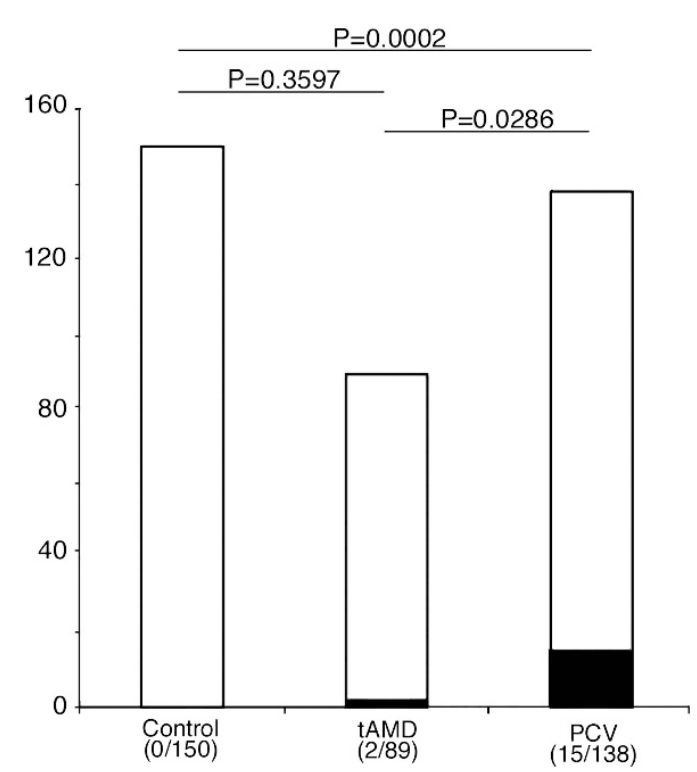

Figure 1 Frequency of a history of central serous chorioretinopathy (CSC) in the control subjects, patients with typical agerelated macular degeneration, and those with polypoidal choroidal vasculopathy in all the enrolled subjects $(n=377)$. $P$-values are the results of post hoc Marascuilo procedure between the two indicated groups after ANOVA. The black and white parts in each bar indicate the proportions of subjects who did and did not have a history of CSC, respectively.

in patients with PCV than in control subjects. In contrast, CSC was not apparently associated with the development of tAMD.

The development of AMD as a sequela of CSC has been studied. Yap and Robertson ${ }^{18}$ reported 38 patients with CSC who were followed up for 11-15 years. Bujarborua ${ }^{19}$ reported on five patients with CSC who were followed up for 7-23 years. Neither of the studies identified an association between a history of CSC and the development of AMD. These studies were valuable because a prospective observation of CSC patients could be extremely powerful evidence regarding the causative role of CSC in the development of PCV. However, there were critical limitations of these studies. First, numbers of the enrolled patients may have been extremely small 
and the follow-up periods may have been extremely short to identify or refute any associations. Second, because the enrolled subjects were predominantly

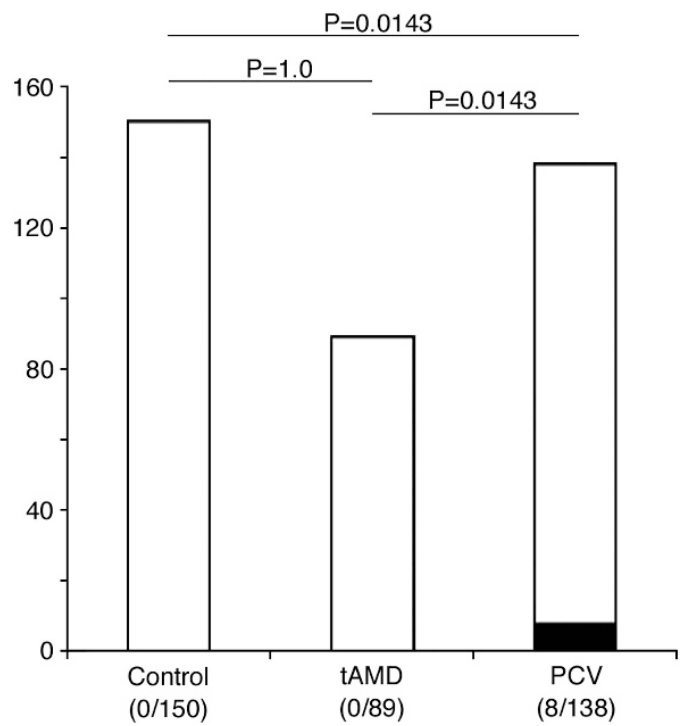

Figure 2 Frequency of funduscopic findings indicating a history of central serous chorioretinopathy (ie, atrophic retinal pigment epithelial tracts and focal photocoagulation scars in the posterior fundus) in the control subjects, patients with typical age-related macular degeneration, and those with polypoidal choroidal vasculopathy in all the enrolled subjects $(n=377)$ $P$-values are the results of post hoc Marascuilo procedure between the two indicated groups after ANOVA. The black and white parts in each bar indicate the proportions of subjects who did and did not have such characteristic findings on funduscopy, respectively.
Caucasians, it would be difficult to address the issue. In contrast, Ahuja et $a l^{20}$ and Yannuzzi et al ${ }^{21}$ discussed PCV as a possible sequela following chronic CSC based on their clinical experiences. Spaide et $a l^{22}$ and Loo et $a l^{23}$ also reported on patients with CSC who concurrently developed choroidal neovascularizaion or during the follow-up. Moreover, other studies have revealed that CSC and PCV have similar characteristics in the choroid. The choroidal hyperpermeability on ICGA was a common feature for both $\mathrm{CSC}^{24,25}$ and PCV. ${ }^{14}$ The choroid in the eyes of patients with CSC and those of patients with PCV was thicker than that of normal patient eyes or in eyes of patients with tAMD. 10,11,26 Koizumi et al ${ }^{14}$ recently identified a history of CSC in $12.4 \%$ patients with PCV and Park et al ${ }^{15}$ reported that $5.3 \%$ patients with PCV had findings of chronic CSC, including an atrophic RPE tract and multiple RPE atrophies, both of which were consistent with our results. The present study showed that CSC could be a predisposing factor for the development of PCV because the frequency of a history of CSC was considerably higher in PCV patients than in control subjects.

We further elucidated the association of a history of CSC and exudative AMD from the viewpoint of their laterality. The laterality of the eyes for the two disorders matched in 9 of 10 patients with both CSC and AMD in one eye. This result formed an interesting background for investigation into the mechanisms that could connect the two disorders. If genetic factors were the most influential, the laterality of CSC and AMD would have been rather random. The matched laterality of CSC and AMD could

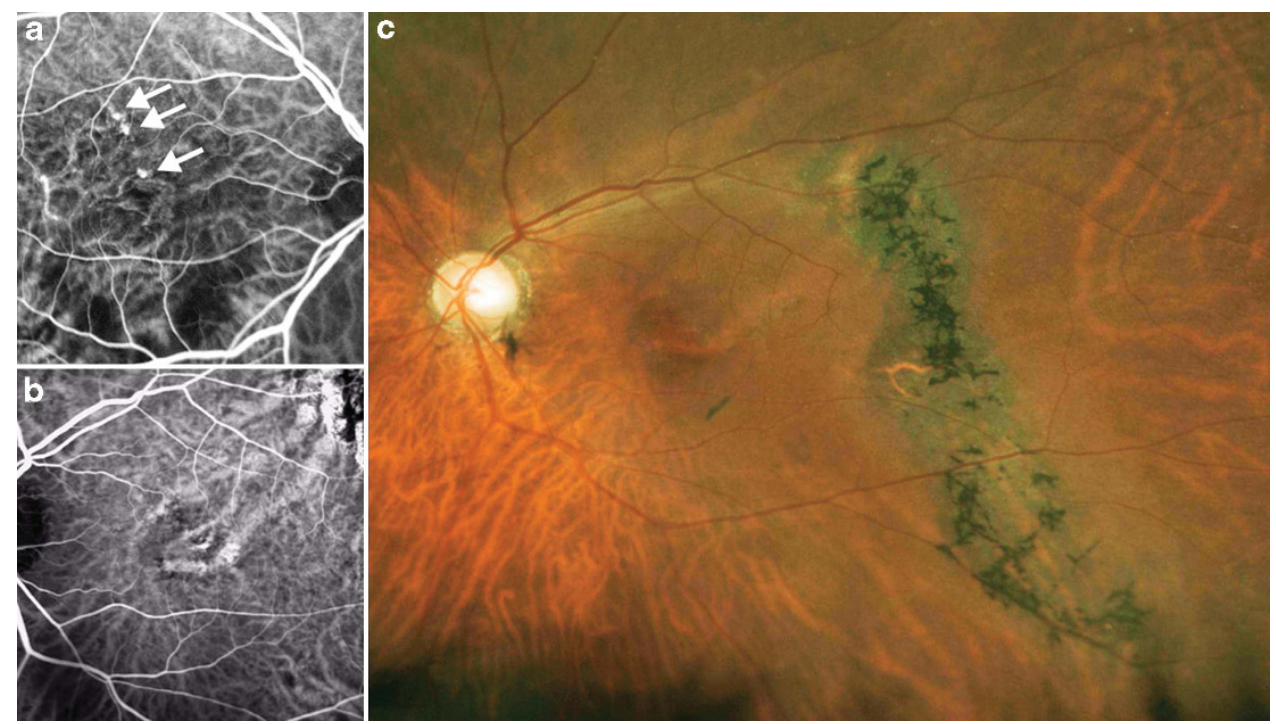

Figure 3 An example case of polypoidal choroidal vasculopathy with an atrophic retinal pigment epithelial (RPE) tract. This patient reported a history of CSC in both eyes. (a) In the right macula, polypoidal choroidal vascular lesions were observed in the inner choroidal vasculature. (b) In the left macula there were dilated choroidal vessels without polypoidal lesions. (c) In the left fundus there was an atrophic RPE tract. 
indicate that the association of the two disorders was not largely genetic, but rather due to RPE atrophy or persistent choroidal abnormality after CSC episodes.

In the present study, we carefully examined the history of CSC history in control subjects and patients with exudative AMD. A history of CSC implied CSC in either eye because there was often a bilateral pathology in the choroidal vasculature even with a unilateral clinical presentation. ${ }^{24,25,27,28}$ Because PCV masquerading as $\mathrm{CSC}^{21}$ was a well-known phenomenon, we had to confirm the reported history of CSC and that these incidences were not actually PCV or exudative AMD. Therefore, in this study, we excluded two reports on the history of CSC that supposedly occurred after the age of 50 years and within 10 years of the onset of AMD. As a result, the CSC episodes that were confirmed in the present study had occurred at the age of 45 years old or less in 14 of the 17 patients. It was extremely unlikely that tAMD or PCV would occur at the age of 45 years or less. In contrast, the misdiagnosis of CSC as AMD was not likely because ICGA had been conducted for all patients with exudative AMD. Furthermore, we carefully addressed the possible confounders, including smoking, hypertension, and systemic steroid use.

Although the prevalence of a history of CSC has not been well understood in the general population, the annual incidence of CSC in Minnesota, USA, had been reportedly as low as 9.9 and 1.7 per 100000 for males and females, respectively. ${ }^{29}$ Even though the prevalence of CSC had not been addressed in the Japanese or Asian population and may be higher than that in the Caucasian population, the prevalence of a history of CSC in patients with PCV reported in the present study or by others ${ }^{14,15}$ was remarkably high compared with that in the general population.

In addition to the careful evaluation through the history, we evaluated more objective findings in the fundus, as conducted in our previous study. ${ }^{13}$ The evaluation of fundus findings also indicated that a history of CSC was more common in patients with PCV than in control subjects.

Several limitations were involved in the current study. Recall bias was possible because most of the reported episodes of CSC had occurred several decades previously. The positive response rate for the history of CSC may be lower than the true rate. In addition, although the patients remembered whether they had a history of CSC, it was difficult for them to recall details about the CSC episodes, including their duration and number of attacks. However, we attempted to prevent the misdiagnosis of AMD for CSC because we performed both fluorescein angiography and ICGA for the accurate diagnosis of subtypes of AMD. The sample population was another limitation because the current study was conducted in a university hospital, which may have led to the inclusion of patients with more severe spectrum of exudative AMD compared with those in the general population. The sample size was sufficiently large to detect the causative effect of a history of CSC for the development of PCV. However, a larger sample size would be necessary to evaluate the relationship between the history of CSC and the development of tAMD.

In conclusion, we showed that the history of CSC could facilitate the development of PCV in the Japanese population. The possible association should be explained to the patients with CSC because they may need to be carefully monitored for the development of exudative AMD or PCV.

\section{Summary}

\section{What was known before}

- History of central serous chorioretinopathy (CSC) has been recently identified and confirmed to be more prevalent in patients with polypoidal choroidal vasculopathy (PCV) compared with typical age-related macular degeneration.

What this study adds

- This study adds a fact that history of CSC is more common in patients with PCV compared with control subjects, which indicates a predisposing role of CSC for the development of PCV.

\section{Conflict of interest}

The authors declare no conflict of interest.

\section{Author contributions}

Design of the study (TU, YN); conduct of the study $(\mathrm{KO}, \mathrm{TT})$, writing the manuscript (TU, KO, TT); critical revision (YN).

\section{References}

1 Attebo K, Mitchell P, Smith W. Visual acuity and the causes of vision loss in Australia: the Blue Mountain Eye Study. Ophthalmology 1996; 103: 357-364.

2 Yuzawa M, Tamakoshi A, Kawamura T, Ohno Y, Uyama M, Honda T. Report on the nationwide epidemiological survey of exudative age-related macular degeneration in Japan. Int Ophthalmol 1997; 21: 1-3.

3 Maruko I, Iida T, Saito M, Nagayama D, Saito K. Clinical characteristics of exudative age-related macular degeneration in Japanese patients. Am J Ophthalmol 2007; 144: 15-22.

4 Yannuzzi LA, Sorenson J, Spaide RF, Lipson B. Idiopathic polypoidal choroidal vasculopathy (IPCV). Retina 1990; 10: $1-8$. 
5 Uyama M, Wada M, Nagai T, Matsubara T, Matsunaga H, Fukushima I et al. Polypoidal choroidal vasuculopathy: natural history. Am J Ophthalmol 2002; 133: 639-648.

6 Yannuzzi LA, Ciardella A, Spaide RF, Rabb M, Freund KB, Orlock DA. The expanding clinical spectrum of idiopathic polypoidal choroidal vasculopathy. Arch Ophthalmol 1997; 115: 478-485.

7 Yannuzzi LA, Wong DW, Sforzolini BS, Goldbaum M, Tang KC, Spaide RF et al. Polypoidal choroidal vasuculopathy and neovasuclarized age-related macular degeneration. Arch Ophthalmol 1999; 117: 1503-1510.

8 Gomi F, Ohji M, Sayanagi K, Sawa M, Sakaguchi H, Oshima Y et al. One-year outcomes of photodynamic therapy in age-related macular degeneration and polypoidal choroidal vasculopathy in Japanese patients. Ophthalmology 2008; 115: 141-146.

9 Gomi F, Sawa M, Sakaguchi H, Tsujikawa M, Oshima Y, Kamei $\mathrm{M}$ et al. Efficacy of intravitreal bevacizumab for polypoidal choroidal vasculopathy. Br J Ophthalmol 2008; 92 70-73.

10 Chung SE, Kang SW, Lee JH, Kim YT. Choroidal thickness in polypoidal choroidal vasculopathy and exudative age-related macular degeneration. Ophthalmology 2011; 118: 840-845.

11 Rishi P, Rishi E, Mathur G, Raval V. Ocular perfusion pressure and choroidal thickness in eyes with polypoidal choroidal vasculopathy, wet-age-related macular degeneration, and normals. Eye 2013; 27(9): 1038-1043.

12 Lima LH, Schubert C, Ferrara DC, Merriam JE, Imamura $Y$ Freund $\mathrm{KB}$ et al. Three major loci involved in age-related macular degeneration are also associated with polypoidal choroidal vasculopathy. Ophthalmology 2010; 117: 1567-1570.

13 Ueta T, Obata R, Inoue Y, Iriyama A, Takahashi H, Yamaguchi T et al. Background comparison of typical age-related macular degeneration and polypoidal choroidal vasculopathy in Japanese patients. Ophthalmolgy 2009; 116: 2400-2406.

14 Koizumi H, Yamagishi T, Yamazaki T, Kinoshita S. Relationship between clinical characteristics of polypoidal choroidal vasculopathy and choroidal vascular hyperpermeability. Am J Ophthalmol 2013; 115: 305-313.

15 Park HS, Kim IT. Clinical characteristics of polypoidal choroidal vasculopathy associated with chronic central serous chorioretionopathy. Korean J Ophthalmol 2012; 26: 15-20.

16 Klais CM, Ober MD, Ciardella AP, Yannuzzi LA. Central serous chorioretinopathy. In: Ryan SJ, Hinton DR, Schachat AP,
Wilkinson CP (eds) Retina: Medical Retina. 4th edn, vol. 2 Elsevier Mosby: Philadelphia, 2006, pp 1135-1161.

17 Haimovici R, Koh S, Gagnon DR, Lehrfeld T, Wellik S. Central Serous Chorioretinopathy Case-Control Study Group. Risk factors for central serous chorioretinopathy: a case-control study. Ophthalmology 2004; 111: 244-249.

18 Yap EY, Robertson DM. The long-term outcome of central serous chorioretinopathy. Arch Ophthalmol 1996; 114: 689-692.

19 Bujarborua D. Long-term follow-up of idiopathic central serous chorioretinopathy without laser. Acta Ophthalmol Scand 2001; 79: 417-421.

20 Ahuja RM, Downes SM, Stanga PE, Koh AH, Vingerling JR, Bird AC. Polypoidal choroidal vasculopathy and central serous chorioetinopathy[letter]. Ophthalmology 2001; 108: 1009-1010.

21 Yannuzzi LA, Freund KB, Goldbaum M, Scassellati-Sforzolini B, Guyer DR, Spaide RF et al. Polypoidal choroidal vasculopathy masquerading as central serous chorioretinopathy. Ophthalmology 2000; 107: 767-777.

22 Spaide RF, Campeas L, Haas A, Yannuzzi LA, Fisher YL, Guyer DR et al. Central serous chorioretinopathy in younger and older adults. Ophthalmology 1996; 103: 2070-2079.

23 Loo RH, Scott IU, Flynn Jr HW, Gass JD, Murray TG, Lewis ML et al. Factors associated with reduced visual acuity during long-term follow-up of patients with idiopathic central serous chorioretinopathy. Retina 2002; 22: 19-24.

24 Prunte C, Flammer J. Choroidal capillary and venous congestion in central serous chorioretinopathy. Am J Ophthalmol 1996; 121: 26-34.

25 Iida T, Kishi S, Hagimura N, Shimizu K. Persistent and bilateral choroidal vascular abnormalities in central serous chorioretinopathy. Retina 1999; 19: 508-512.

26 Maruko I, Iida T, Sugano Y, Ojima A, Ogasawara M, Spaide RF. Subfoveal choroidal thickness after treatment of central serous chorioretinopathy. Ophthalmology 2010; 117: 1792-1799.

27 Spaide RF, Hall L, Hass A, Campeas L, Yannuzzi LA, Fisher YL et al. Indocyanine green videoangiography of older patients with central serous chorioretinopathy. Retina 1996; 16: 203-213.

28 Kim YT, Kang SW, Bai KH. Choroidal thickness in both eyes of patients with unilaterally active central serous chorioretinopathy. Eye 2011; 25: 1635-1640.

29 Kitzmann AS, Pulido JS, Diehl NN, Hodge DO, Burke JP. The incidence of central serous chorioretinopathy in Olmsted County, Minnesota, 1980-2002. Ophthalmology 2008; 115: 169-173. 